\title{
Results of the Saclay NaI(Tl) WIMP Search Experiment and Comparison with other $\mathrm{NaI}(\mathrm{Tl})$ Experiments
}

\author{
G. Gerbier ${ }^{1}$, J. Mallet ${ }^{1}$, L. Mosca ${ }^{1}$, and C. Tao $^{2}$ \\ 1 DSM/DAPNIA/SPP, C.E.A. Saclay, F-91191 Gif-sur-Yvette, France \\ 2 CPPM, IN2P3/CNRS and Université Aix-Marseille II, 163 av. de Luminy, case 907, \\ F-13288 Marseille Cedex 09, France
}

\begin{abstract}
Underground data for WIMP search have been obtained from a 83 days rum of a $10 \mathrm{~kg} \mathrm{NaI}(\mathrm{Tl})$ detector in a low activity environment at the Laboratoire Souterrain de Modane (LSM). Emphasis is put in this paper on the understanding of the $2-5 \mathrm{keV}$ energy region, crucial both at establishing limits and at analyzing annual modulation of Spin Independent WIMP interactions. Comparison is done with other experiments. Would there be a signal in DAMA data, the residual electromagnetic background energy spectrum exhibits a strange shape. Pulse shape analysis at higher energies have shown an unexpected population of "U" events, also observed by another experiment at the same rate. Additional data obtained at LSM on "U" events are reported. A possible interpretation of these events is related to alpha decays of Radon descendants near the surface of the crystal.
\end{abstract}

\section{Introduction}

The results of the Saclay/Lyon groups NaI(Tl) experiment performed at the Laboratoire Souterrain de Modane have been recently published in [1]. The goal of this contribution is first to give the main findings of this experiment, namely about the shape of the energy spectrum in the $2-10 \mathrm{keV}$ region and about the identification of an unexpected population of "Bump/U" events in the 10-70 $\mathrm{keV}$ region. Secondly, a short comparison of performances and findings with other $\mathrm{NaI}(\mathrm{Tl})$ experiments is done, in particular in view of the understanding of an important claim concerning the DAMA annual modulation signal [2].

\section{Set-Up}

The setup used at LSM consisted of a $10 * 10 * 25 \mathrm{~cm}$ long $\mathrm{NaI}(\mathrm{Tl})$ low activity crystal viewed by two EMI 3 inch low activity PMT through $10 \mathrm{~cm}$ quartz light guides. The detector was installed inside a very low activity ancient lead shield enclosed in a plexiglass box continuously flushed with nitrogen to avoid radon contamination. The measured photoelectron yield was about 7 pe/keV. All pulses above $1.8 \mathrm{keV}$ were digitised at $200 \mathrm{Mhz}$ and recorded on tape. The live time data taking was 83 days. 
This detector module is very similar to the ones used in the DAMA experiment in size, crystal growing method, manufacturer, housing, light guides and PMT type [3].

\section{Low Energy Spectrum}

On figure 1 is shown the obtained raw differential energy rate of a subsample of the total exposure (open circles).

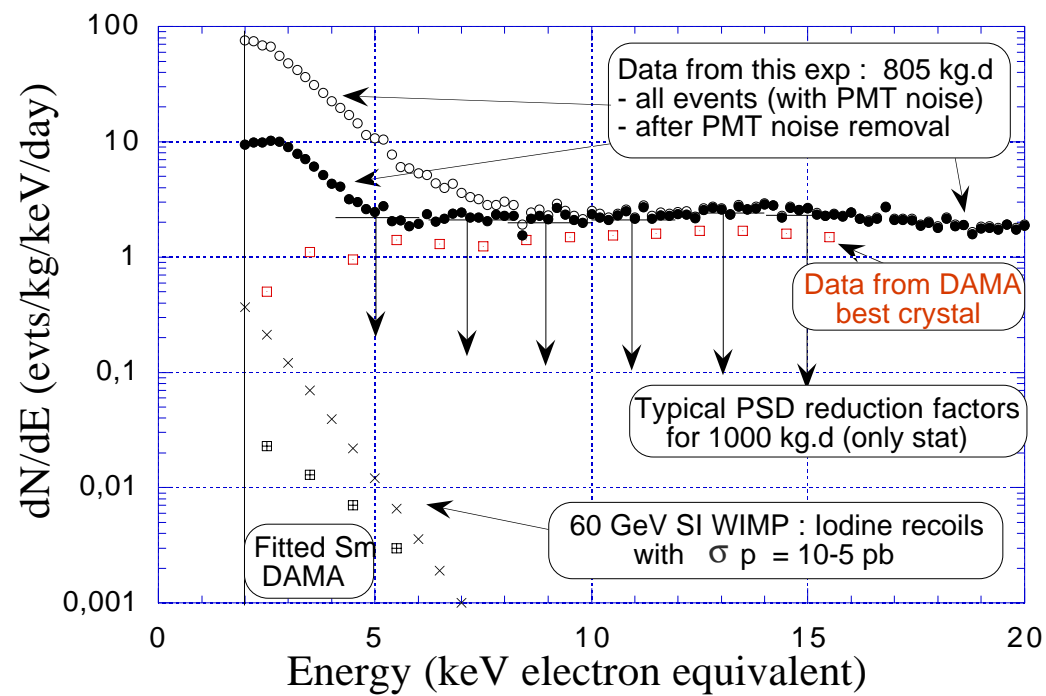

Fig. 1. Energy spectra of a) expected signal in NaI(Tl) from a $60 \mathrm{GeV} \mathrm{SI} \mathrm{WIMP,} \mathrm{b)} \mathrm{of}$ the experimental spectra measured by this experiment (raw and cleaned up) and the DAMA one of their best crystal, together with typical reduction factors obtained with PSD.

The 2 to $8 \mathrm{keV}$ energy region is dominated by PMT noise pulses. The total rate in the $2-3 \mathrm{keV}$ bin goes up to $80 \mathrm{evts} / \mathrm{kg} / \mathrm{keV} /$ day. These pulses have characteristic shapes, much sharper than scintillation light pulses. They can be clearly identified on the figure 2 which shows a scatter diagram of a "sharpness" parameter as a function of energy for all the recorded pulses.

By applying adequate cuts on this parameter and on the asymetry between the two PMT pulse heights, PMT noise can be mostly rejected. We found that we keep $0.5 \%$ of PMT noise in the $2-3 \mathrm{keV}$ bin, that is $0.4 \mathrm{evts} / \mathrm{kg} / \mathrm{keV} /$ day. On the other hand the efficiency for keeping the good scintillation pulses is 75 $\%$ in this same bin of energy and rises up to $99 \%$ at $4 \mathrm{keV}$.

The spectrum cleaned up for noise and corrected for the efficiency is shown on figure 1 (black circles). The rising up of the spectrum at the lowest energy, up to $10 \mathrm{evts} / \mathrm{kg} / \mathrm{kev} /$ day at $2.5 \mathrm{keV}$ is due to true $\mathrm{NaI}(\mathrm{Tl})$ pulses as shown by 


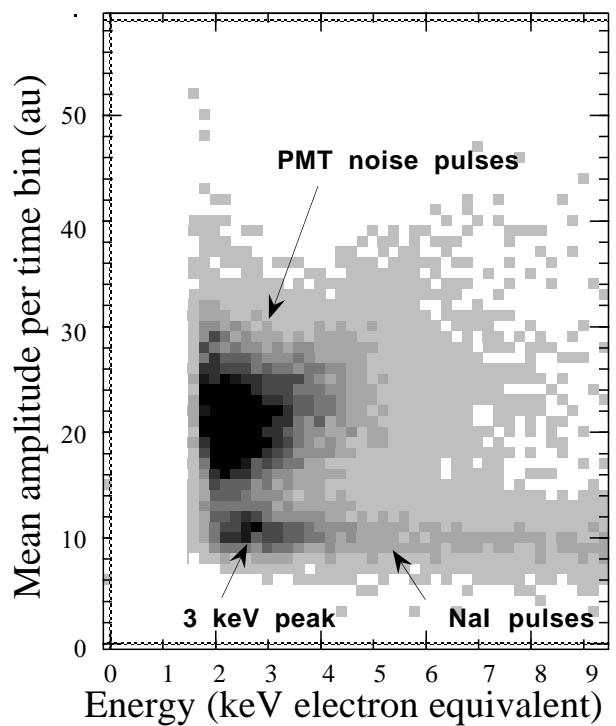

Fig. 2. Scatter plot of the mean amplitude per time bin as a function of energy for data events before appyling any software cuts. The $3 \mathrm{keV}$ peak can clearly be seen in the $\mathrm{NaI}(\mathrm{Tl})$ pulses region

the darkening of the $\mathrm{NaI}(\mathrm{Tl})$ region on figure 2 in the $2-4 \mathrm{keV}$ region. It is not due to badly rejected PMT noise.

\subsection{Low energy spectra from other experiments}

Energy spectra obtained by other experiments using NaI(Tl) (UKDMC [4], Saragoza/Canfranc [5], Elegants V [6]) are shown on figure 3. The absolute rates are high for Elegants $\mathrm{V}$ and Saragoza/Canfranc but comparable to our result for UKDMC though at a higher energy threshold. All spectra show an increase of rate at low energies.

However, the spectra observed by the DAMA group [2] for their different crystals show a rather flat shape in the 3 to $10 \mathrm{keV}$ region and even a drop towards low energy from the $3.5 \mathrm{keV}$ to the $2.5 \mathrm{keV}$ bin. The rate for the best crystal even lowers to $0.5 \mathrm{evts} / \mathrm{kg} / \mathrm{keV} /$ day at $2.5 \mathrm{keV}$ (see open squares on figure 1 ), that is a factor 20 less than the presently measured rate. The rate above 10 $\mathrm{keV}$ is roughly at the same level as the present data.

This is rather surprising as one expects that the origin of most events at low energy are Compton interactions of higher energy gamma rays from radioactive nucleides either from inside or from outside the detector. The expected shape in this energy region is a smooth variation with energy but not a sudden drop of a factor two within a two $\mathrm{keV}$ range. 

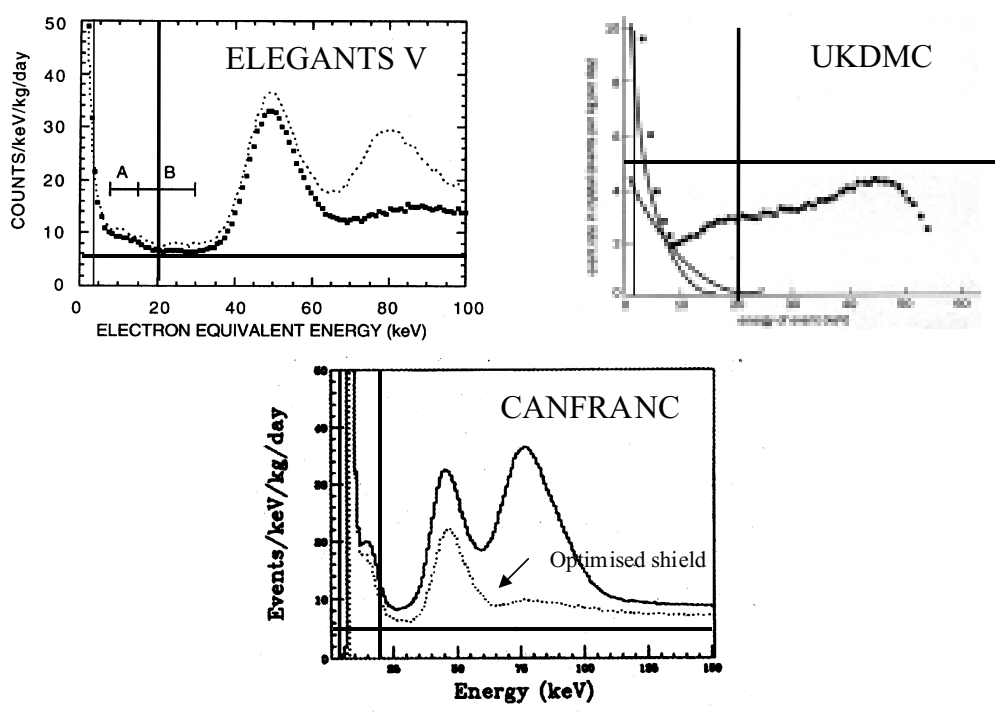

Fig. 3. Energy spectra of UKDMC, Saragoza-Canfranc, ELEGANTS NaI(Tl) experiments, the black horizontal line corresponds to $5 \mathrm{evts} / \mathrm{kg} / \mathrm{keV} /$ day, the vertical one to $20 \mathrm{keV}$

Consequences on Exclusion plot. As noted in [7], Spin Independent (SI) coupling WIMP interactions are expected to occur mostly on Iodine nuclei at visible energies lower than $7 \mathrm{keV}$ (see expected shape of a $60 \mathrm{GeV}$ WIMP signal on figure 1). The sensitivity is given by the maximum amount of signal which can be accomodated within the data. From figure 1, it can be seen that the DAMA spectrum has a better sensitivity in cross section than the present one, by a factor 20 , because of the low rate of the $2.5 \mathrm{keV}$ data point.

Pulse Shape Discrimination (PSD) which allows to discriminate between the electron induced (background) and nuclear recoil induced pulses (signal) should in principle produce an increase of the sensitivity by reducing the allowed residual rate of nuclear recoils. However this analysis is possible only above $4 \mathrm{keV}$ electron equivalent energy. Given the DAMA exposure [2], it has been shown in [8] that the PSD at energies higher than $4 \mathrm{keV}$ does not help improving the sensitivity relative to the use of the rate at $2.5 \mathrm{keV}$.

This mostly explains the difference in sensitivity of the two experiments in the cross section mass exclusion diagram for SI WIMP : of about $1310^{-5} \mathrm{pb}$ for this experiment as compared to $0.510^{-5} \mathrm{pb}$ for DAMA at a WIMP mass of 100 $\mathrm{GeV}$. 
Consequences on annual modulation signal This strange feature of the DAMA energy spectrum becomes even stronger if there is a WIMP signal in the data. As stated in ref [2], the detected modulated signal Sm leads to an absolute level of WIMP signal S0 in the total energy spectrum at the level of 0.4 to 0.5 evts $/ \mathrm{kg} / \mathrm{keV} /$ day in the $2-3 \mathrm{keV}$ bin, while the mean (lowest crystal $\mathrm{C} 8$ ) total rate is $1.0(0.5)$ evts $/ \mathrm{kg} / \mathrm{keV} /$ day. This means that the residual electromagnetic background shows an even bigger drop at the lowest energy, $0.5 \pm 0.1(0.0 \pm$ 0.1 ) evts $/ \mathrm{kg} / \mathrm{keV} /$ day for the mean ("best") rate on all the crystals. This point is not discussed in the published DAMA paper.

There are other points which cast doubt on the overall consistency of a real signal in the DAMA data :

- the inconsistency between the signals given by the various modules of the detector in the first report [8]. It has been shown [9] that the signal was present in 3 crystals out of 9 .

- the "stability" of the significance of the result with statistics. The $4 \sigma$ result with a $4549 \mathrm{~kg}$.day statistics from the first report [8] stays a $4 \sigma$ signal in the last $57986 \mathrm{~kg}$.day statistics [2] with the same $7.610^{-5} \mathrm{pb}$ signal.

- the presentation of the modulated signal with time [2]. There are many knobs to play with to enrich a possible modulated signal: energy window, selection of time width bin. It can be noticed on the modulation plot [2] that the binning varies from 1 to 3 months. Also, this plot does not show the dead time of the experiment (of about $38 \%$ ).

\section{Pulse Shape Analysis and U Event Population}

Pulse shape analysis of the $805 \mathrm{~kg}$.day data, at energies higher than $10 \mathrm{keV}$, have shown an unexpected population of "U" events with even faster decay times than nuclear recoil pulses [1]. UKDMC has observed the same kind of events, the "Bump" events [4] with similar total rates of about 3 evts $/ \mathrm{kg} /$ day and differential energy spectra (see figure 4).

These events have not been observed in the DAMA experiment. The Elegants $\mathrm{V}$ and Saragoza/Canfranc experiments, where the pulse shape information was not recorded, could not give any information on these events.

Two other $10 \mathrm{~kg}$ crystals, manufactured by BICRON twelve years ago, have been recently measured at LSM. Measured U event spectra are shown also on figure 4 . They are again very similar to the ones previously obtained.

The most likely explanation consists in a radon surface contamination. Recoiling nuclei from descendants of radon implant slightly inside the crystal (by about $0.1 \mu \mathrm{m}$ ). Subsequent alpha decays give rise to recoiling nucleus and escaping $\mathrm{MeV}$ alpha depositing their energy close to the surface.

Calculations by the UK group [10] indeed give the right energy spectrum but show that the needed amount of radon accumulated at the surface between the cutting and the sealing of the crystal is much higher than what can be reasonably inferred from the known way of manufacturing low activity detectors. 


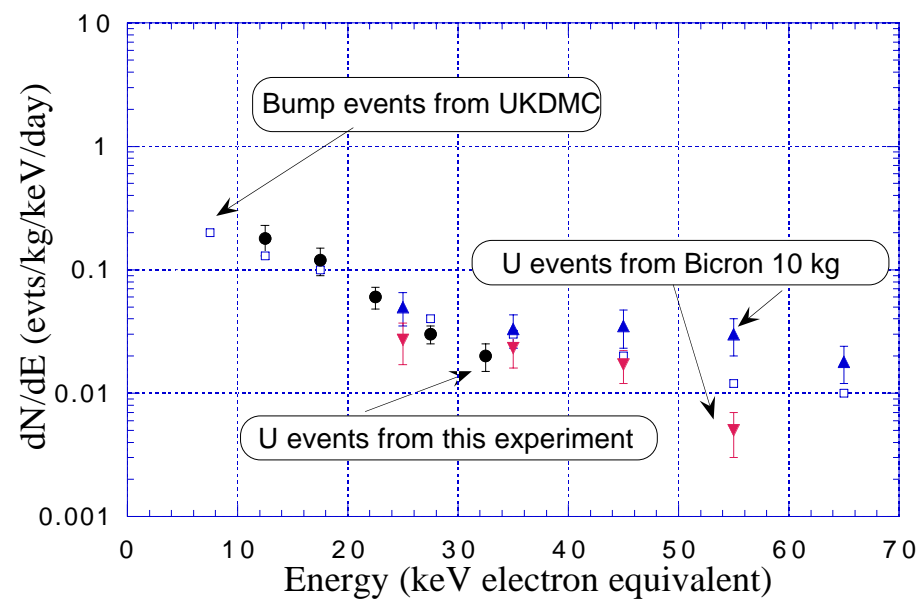

Fig. 4. Energy spectra of U events (from this experiment), Bump events from UKDMC result [4], and more recent results from Bicron crystals measured at LSM

To disentangle this issue, the UKDMC group and Saclay group have led together a recent experimental investigation along two lines :

- The crystal used in the present experiment has been also measured at Boulby mine, and was found to give similar energy spectrum as in LSM, and similar rates of $U$ events. This confirms that the $U$ events are indeed due to the detector.

- Tests of scraping the surface have also been performed by the UK group and lead to a substantial decrease of the U events. This confirms the original hypothesis of surface contamination though it does not account for the surprising similarity of spectra between crystals of very different origins and manufacturers.

The hope is to suppress the U/Bump events and then to restaure the initial potential sensitivity of NaI to WIMP signal.

\section{References}

1. G. Gerbier et al.: Astropart. Phys. 11, 287 (1999)

2. R. Bernabei et al.: Phys. Lett. B 450, 448 (1999) and Phys. Lett. B 480, 23 (2000)

3. R. Bernabei et al., INFN/AE-1998/23

4. P.F. Smith et al.: Phys. Rep. 307, 275 (1998)

5. E. Garcia et al.: Phys. Rev. D51, 1458 (1995)

6. K. Fushimi et al.: Astropart. Phys. 12, 185 (1999)

7. G. Gerbier et al.: Proc. of DM2000 Symposium, Marina Del Rey (Feb 2000)

8. R. Bernabei et al.: Phys. Lett. B 424, 195 (1998)

9. G. Gerbier et al.: astro-ph 9710181 and astro-ph 9902194

10. N.J.T. Smith et al: Phys. Lett. B 485, 9 (2000) 